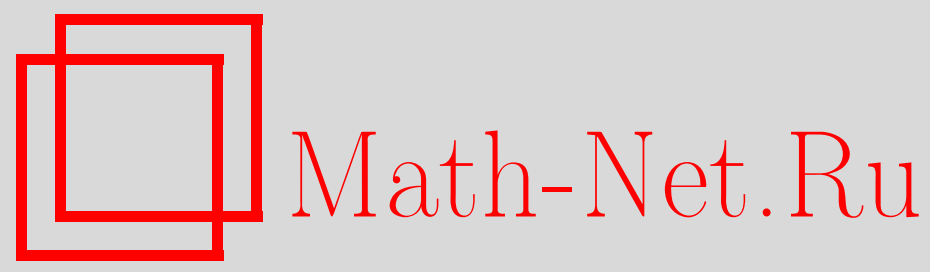

А. Н. Назарова, Нормальная аппроксимация для случайных линейных процессов и полей в гильбертовом пространстве, Матем. заметки, 2000, том 68, выпуск 3, 421-428

DOI: https://doi.org/10.4213/mzm959

Использование Общероссийского математического портала Math-Net.Ru подразумевает, что вы прочитали и согласны с пользовательским соглашением http://www.mathnet.ru/rus/agreement

Параметры загрузки:

IP: 3.93 .64 .190

26 апреля 2023 г., $11: 10: 11$

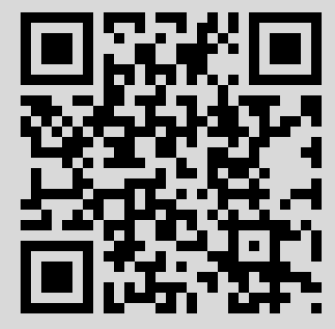




\section{НОРМАЛЬНАЯ АППРОКСИМАЦИЯ ДЛЯ СЛУЧАЙНЫХ ЛИНЕЙНЫХ ПРОЦЕССОВ И ПОЛЕЙ В ГИЛЬБЕРТОВОМ ПРОСТРАНСТВЕ}

\section{А. Н. Назарова}

Доказана центральная предельная теорема для линейных случайных полей, заданных на целочисленной решетке произвольной размерности и принимающих значения в гильбертовом пространстве. Показано, что предложенные условия оптимальны.

Библиография: 6 названий.

Исследование предельного поведения сумм случайных величин со значениями в банаховых или гильбертовых пространствах - традиционная область теории вероятностей, имеющая ряд статистических приложений. В данной работе изучаются суммы, порожденные линейными случайными полями со значениями в гильбертовом пространстве. Такие суммы возникают в задачах оценивания и прогнозирования для некоторых классов случайных процессов и полей (см., например, [1]). Цель данной работы - доказать центральную предельную теорему (ЦПТ) для линейных полей, заданных на целочисленной решетке $\mathbb{Z}^{d}$ при произвольном $d \geqslant 1$. Тем самьг, достигается обобщение результатов недавней статьи [2]. Доказано также, что предложенные условия носят оптимальньй характер.

Нам потребуется ряд определений.

Пусть $H$ - сепарабельное действительное гильбертово пространство, его норма $\|\cdot\|_{H}$ индуцирована скалярным произведением $\langle\cdot, \cdot\rangle, \mathscr{L}(H)$ - пространство линейных ограниченных операторов из $H$ в $H$. Пусть $\left\{\xi_{\boldsymbol{k}}, \boldsymbol{k} \in \mathbb{Z}^{d}\right\}$ - поле случайных величин, определенных на вероятностном пространстве $(\Omega, \mathscr{F}, \mathrm{P})$ и принимаюших значения в $H, \mathrm{a}$ $\left\{a_{\boldsymbol{k}}, \boldsymbol{k} \in \mathbb{Z}^{d}\right\}$ - семейство линейных операторов из $\mathscr{L}(H)$.

Линейным процессом (или полем) назьвается множество случайных величин следующего вида:

$$
X_{\boldsymbol{k}}=\sum_{\boldsymbol{j} \in \mathbb{Z}^{d}} a_{\boldsymbol{j}}\left(\xi_{\boldsymbol{k}-\boldsymbol{j}}\right), \quad \boldsymbol{k} \in \mathbb{Z}^{d}
$$

Здесь и далее мы предполагаем, что $\xi_{\boldsymbol{k}}$ независимы, одинаково распределены (н.о.р.), центрированы, $\mathrm{E}\left\|\xi_{\mathbf{0}}\right\|^{2}<\infty$, a $\sum\left\|a_{\boldsymbol{j}}\right\|^{2}<\infty$. Тогда нетрудно видеть (см. [3, гл. 3.2]), что ряд (1) сходится почти наверное и в пространстве $\mathscr{L}_{2}(\Omega)$, состоящем из функций, отображающих $\Omega$ в $H$ и имеющих интегрируемьй квадрат нормы.

Определим частичные суммы

$$
S_{n}=\sum_{1 \leqslant k \leqslant n} X_{k}, \quad n \geqslant 1 .
$$


Для векторов $\boldsymbol{k}=\left(k_{1}, \ldots, k_{d}\right)$ и $\boldsymbol{n}=\left(n_{1}, \ldots, n_{d}\right)$ неравенство $\boldsymbol{k} \leqslant \boldsymbol{n}$ означает, что $k_{i} \leqslant n_{i}$ при всех $i=1, \ldots, d$.

В простейшем случае, когда $\xi_{k}$ - действительнозначные случайные величины, $a_{k}-$ действительные числа, а индекс $k \in \mathbb{Z}$, т.е. $d=1$, поведение $S_{n}$ хорошо изучено. (Для таких величин вместо $a_{j}\left(\xi_{k-j}\right)$ в (1) берется $a_{j} \xi_{k-j}$.) Например, известна следующая

Теорема 1 (см. [4, теорема 18.6.5]). Пусть $\left\{\xi_{k}\right\}_{k \in \mathbb{Z}}$ - н.о.р. центрированнье случайные величины с конечным вторым моментом, $\left\{a_{k}\right\}_{k \in \mathbb{Z}}$ - последовательность действительных чисел таких, что

$$
\sum_{k=-\infty}^{\infty}\left|a_{k}\right|^{2}<\infty
$$

a $X_{k}, S_{n}$ определены выше. Тогда если $\mathrm{E}\left|S_{n}\right|^{2} \rightarrow \infty, n \rightarrow \infty$, то

$$
\frac{S_{n}}{\sqrt{\mathrm{E}\left|S_{n}\right|^{2}}} \stackrel{\mathscr{D}}{\rightarrow} \mathcal{N}(0,1)
$$

В случае, если $\xi_{k}$ принимают значения в бесконечномерном пространстве, ситуация существенно меняется. Так, в теореме 3 из работы [2] было показано, что вьполнение условия (2) (конечно, в нем надо заменить $\left|a_{k}\right|$ на $\left\|a_{k}\right\| \mathscr{L}(H)$ ) не обеспечивает даже плотности последовательности распределений $S_{n} / \sqrt{\mathrm{E}\left|S_{n}\right|^{2}}$.

Для вьполнения ЦПТ с нормировкой $\sqrt{n}$ оказьвается достаточньм (см. [2, теорема 2]) более сильное условие

$$
\sum_{k=-\infty}^{\infty}\left\|a_{k}\right\|_{\mathscr{L}(H)}<\infty
$$

В данной работе ЦПТ доказана для случайных величин, принимающих значения в гильбертовом пространстве и индексированных многомерным индексом $\boldsymbol{k} \in \mathbb{Z}^{\boldsymbol{d}}$ (теорема 2). Достаточное условие оказывается аналогичным (3). Опираясь на идеи работы [2], показано, что полученное условие неулучшаемо (теорема 3 ).

Для любого вектора $\boldsymbol{n}=\left(n_{1}, \ldots, n_{d}\right) \in \mathbb{N}^{d}$ положим $|\boldsymbol{n}|=n_{1} \cdots n_{d}$.

Теорема 2. Пусть $\left\{\xi_{\boldsymbol{k}}, k \in \mathbb{Z}^{d}\right\}$ - описанное выше случайное поле. Предположим, чmo

$$
\sum_{j \in \mathbb{Z}^{d}}\left\|a_{\boldsymbol{j}}\right\|<\infty
$$

Tогда

$$
\frac{1}{\sqrt{|\boldsymbol{n}|}} S_{\boldsymbol{n}} \stackrel{\mathscr{D}}{\rightarrow} \mathcal{N}\left(0, A C_{\xi_{0}} A^{*}\right), \quad \boldsymbol{n} \rightarrow \infty,
$$

где $A=\sum_{\boldsymbol{j} \in \mathbb{Z}^{d}} a_{\boldsymbol{j}}, A^{*}-$ сопряженный оператор для $A, C_{\xi_{0}}-$ ковариачионный опеpатор $\xi_{\mathbf{0}}$, a $\boldsymbol{n} \rightarrow \infty$ означает, что $|\boldsymbol{n}| \rightarrow \infty$, причем все координаты не убывают.

Для доказательства понадобится следующий вспомогательный результат. 
ЛЕмма 1. Пусть $\left\{b_{j}\right\}_{j \in \mathbb{Z}^{d}}$ - әлементы какого-либо нормированного пространства - таковы, что

$$
\begin{gathered}
\sum_{\boldsymbol{j} \in \mathbb{Z}^{d}}\left\|b_{\boldsymbol{j}}\right\|<\infty \\
\sum_{\boldsymbol{j} \in \mathbb{Z}^{d}} b_{\boldsymbol{j}}=0 .
\end{gathered}
$$

Тогда

$$
\frac{1}{|n|} \sum_{j \in \mathbb{Z}^{d}}\left\|\sum_{1-j \leqslant i \leqslant n-j} b_{i}\right\|^{2} \rightarrow 0, \quad n \rightarrow \infty .
$$

Условимся запись $[-\boldsymbol{x}, \boldsymbol{x}]$ для любого вектора $\boldsymbol{x}=\left(x_{1}, \ldots, x_{d}\right) \in \mathbb{R}^{d}$ понимать как обозначение прямоугольника в $\mathbb{R}^{d}$ :

$$
[-\boldsymbol{x}, \boldsymbol{x}]=\left[-x_{1}, x_{1}\right] \times \cdots \times\left[-x_{d}, x_{d}\right]
$$

Аналогично

$$
(-\boldsymbol{x}, \boldsymbol{x})=\left(-x_{1}, x_{1}\right) \times \cdots \times\left(-x_{d}, x_{d}\right) .
$$

ДоКАЗАТЕЛЬСТво ЛЕМмы 1. Обозначим

$$
D_{n}=\sum_{j \notin(-n, n)}\left\|b_{j}\right\|
$$

В силу (6) имеем $D_{\boldsymbol{n}} \rightarrow 0$ при $\boldsymbol{n} \rightarrow \infty$. Теперь заметим, что

$$
\begin{aligned}
& \frac{1}{|n|} \sum_{j \notin(-2 n, 2 n)}\left\|\sum_{1-j \leqslant i \leqslant n-j} b_{i}\right\|^{2} \leqslant \frac{1}{|n|} \sum_{j \notin(-2 n, 2 n)}\left(\sum_{1-j \leqslant i \leqslant n-j}\left\|b_{i}\right\|\right)^{2} \\
& \quad \leqslant \frac{1}{|n|} \sum_{j \notin(-2 n, 2 n)}\left(\sum_{1-j \leqslant i \leqslant n-j}\left\|b_{i}\right\|\right)\left(\sum_{i \notin(-n, n)}\left\|b_{i}\right\|\right) \\
& \leqslant D_{n} \cdot \frac{1}{|n|} \sum_{j \in \mathbb{Z}^{d}}\left(\sum_{1-j \leqslant i \leqslant n-j}\left\|b_{i}\right\|\right)=D_{n} \cdot \sum_{j \in \mathbb{Z}^{d}}\left\|b_{j}\right\| \rightarrow 0, \quad n \rightarrow \infty
\end{aligned}
$$

здесь мы снова учли (6).

Рассмотрим функцию

$$
h_{n}(\boldsymbol{x})=\left\|\sum_{1-[|\boldsymbol{n}| \boldsymbol{x}] \leqslant i \leqslant n-[|n| x]} b_{i}\right\|^{2}
$$

для всех $\boldsymbol{x} \in[-\mathbf{2}, \mathbf{2}]$. Здесь [·] означает взятие целой части. Для вектора из $\mathbb{R}^{d}$ целая часть вычисляется покоординатно. Заметим, что из (6) и (7) следует, что $\forall \boldsymbol{x} \neq \mathbf{0}$ $h_{\boldsymbol{n}}(\boldsymbol{x}) \rightarrow 0$ при $\boldsymbol{n} \rightarrow \infty$ и

$$
\left|h_{\boldsymbol{n}}(\boldsymbol{x})\right| \leqslant\left(\sum_{\mathbf{1} \leqslant \boldsymbol{i} \leqslant \boldsymbol{n}} \| b_{\boldsymbol{i}-[|\boldsymbol{n}| \boldsymbol{x}] \|}\right)^{2} \leqslant\left(\sum_{\boldsymbol{i} \in \mathbb{Z}^{d}}\left\|b_{\boldsymbol{i}}\right\|\right)^{2}<\infty .
$$


Тогда

$$
\begin{aligned}
\frac{1}{|\boldsymbol{n}|} \sum_{\boldsymbol{j} \in[-2 \boldsymbol{n}, 2 \boldsymbol{n}-\mathbf{1}]}\left\|\sum_{\mathbf{1}-\boldsymbol{j} \leqslant \boldsymbol{i} \leqslant \boldsymbol{n}-\boldsymbol{j}} b_{\boldsymbol{i}}\right\|^{2} & =\frac{1}{|\boldsymbol{n}|} \sum_{\boldsymbol{j} \in[-2 \boldsymbol{n}, 2 \boldsymbol{n}-\mathbf{1}]} h_{\boldsymbol{n}}\left(\frac{\boldsymbol{j}}{|\boldsymbol{n}|}\right) \\
& =\sum_{\boldsymbol{j} \in[-2 \boldsymbol{n}, 2 \boldsymbol{n}-\mathbf{1}]} \int_{[\boldsymbol{j} /|\boldsymbol{n}|,(\boldsymbol{j}+\mathbf{1}) /|\boldsymbol{n}|]} h_{\boldsymbol{n}}(\boldsymbol{x}) d \boldsymbol{x} \\
& =\int_{[-2 \boldsymbol{n} /|\boldsymbol{n}|, \boldsymbol{n} /|\boldsymbol{n}|]} h_{\boldsymbol{n}}(\boldsymbol{x}) d \boldsymbol{x} \\
& \leqslant \int_{[-\mathbf{2}, \mathbf{2}]} h_{\boldsymbol{n}}(\boldsymbol{x}) d \boldsymbol{x} \rightarrow 0, \quad \boldsymbol{n} \rightarrow \infty,
\end{aligned}
$$

по теореме Лебега о мажорируемой сходимости.

Теперь из (9) и (10) получаем (8). Лемма доказана.

ДоКАЗАТЕЛЬСТВо ТЕОРЕМЫ 2. Сначала покажем, что в условиях этой теоремы выполнено соотношение

$$
\frac{1}{|\boldsymbol{n}|} \mathrm{E}\left\|S_{\boldsymbol{n}}-A \sum_{\mathbf{1} \leqslant \boldsymbol{k} \leqslant \boldsymbol{n}} \xi_{\boldsymbol{k}}\right\|_{H}^{2} \rightarrow 0, \quad \boldsymbol{n} \rightarrow \infty
$$

Действительно,

$$
S_{n}=\sum_{1 \leqslant k \leqslant n} X_{k}=\sum_{1 \leqslant k \leqslant n} \sum_{m \in \mathbb{Z}^{d}} a_{m}\left(\xi_{k-m}\right)=\sum_{j \in \mathbb{Z}^{d}}\left(\sum_{1 \leqslant k \leqslant n} a_{k-j}\right) \xi_{j}
$$

Поэтому

$$
\begin{aligned}
S_{n}-A \sum_{1 \leqslant k \leqslant n} \xi_{k} & =\sum_{1 \leqslant j \leqslant n}\left(\sum_{1 \leqslant k \leqslant n} a_{k-j}-A\right) \xi_{j}+\sum_{j \notin[1, n]}\left(\sum_{1 \leqslant k \leqslant n} a_{k-j}\right) \xi_{j} \\
& =\sum_{j \in \mathbb{Z}^{d}}\left(\sum_{1 \leqslant k \leqslant n} b_{k-j}\right) \xi_{j}
\end{aligned}
$$

где $b_{0}=a_{0}-A$ и $b_{\boldsymbol{i}}=a_{\boldsymbol{i}}$ для всех $\boldsymbol{i} \neq \mathbf{0}$.

Очевидно, операторы $\left\{b_{\boldsymbol{k}}\right\}_{\boldsymbol{k} \in \mathbb{Z}^{d}}$ удовлетворяют условиям леммы 1. Тогда, используя (12), имеем

$$
\frac{1}{|\boldsymbol{n}|} \mathrm{E}\left\|S_{\boldsymbol{n}}-A \sum_{\mathbf{1} \leqslant \boldsymbol{k} \leqslant \boldsymbol{n}} \xi_{\boldsymbol{k}}\right\|_{H}^{2} \leqslant \frac{\mathrm{E}\left\|\xi_{0}\right\|_{H}^{2}}{|\boldsymbol{n}|} \sum_{\boldsymbol{j} \in \mathbb{Z}^{d}}\|\|_{1-\boldsymbol{j} \leqslant \boldsymbol{i} \leqslant \boldsymbol{n}-\boldsymbol{j}} b_{\boldsymbol{i}} \|^{2} \rightarrow 0, \quad \boldsymbol{n} \rightarrow \infty .
$$

Далее, из ЦПТ для н.о.р. центрированных случайных величин со значениями в $H$, имеющих конечньй второй момент (см., например, [5]), получаем

$$
\frac{1}{\sqrt{|n|}} \sum_{1 \leqslant k \leqslant n} \xi_{\boldsymbol{k}} \stackrel{\mathscr{D}}{\rightarrow} \mathcal{N}\left(0, C_{\xi_{0}}\right), \quad \boldsymbol{n} \rightarrow \infty
$$


Поскольку $A$ - линейньй оператор, имеем

$$
\frac{1}{\sqrt{|\boldsymbol{n}|}} A \sum_{\mathbf{1} \leqslant \boldsymbol{k} \leqslant \boldsymbol{n}} \xi_{\boldsymbol{k}} \stackrel{\mathscr{D}}{\rightarrow} \mathcal{N}\left(0, A C_{\xi_{0}} A^{*}\right), \quad \boldsymbol{n} \rightarrow \infty
$$

Теперь из (11) и (13), применяя теорему 4.1 из [6], получим (5). Теорема доказана.

Следующая теорема показывает, что условие (4) теоремы 2 не может быть улучшено, а значит, в случае бесконечномерного пространства не существует никакого аналога теоремы 1.

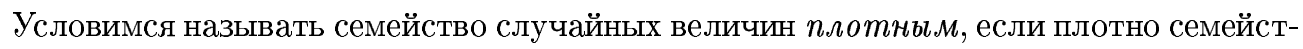
во их распределений.

ТЕОрема 3. Условие (4) теоремы 2 точно, т.е. для любого множества положительных чисел $t_{\boldsymbol{k}}\left(\boldsymbol{k} \in \mathbb{Z}^{d}\right)$ таких, что

$$
\sum_{k \in \mathbb{Z}^{d}} t_{k}=\infty, \quad \sum_{k \in \mathbb{Z}^{d}} t_{k}^{2}<\infty
$$

существует поле н.о.р. иентрированных случайных величин $\left\{\xi_{j}, j \in \mathbb{Z}^{d}\right\}$ со значениями в $H$, для которых $0<\mathrm{E}\left\|\xi_{0}\right\|_{H}^{2}<\infty$, с существует семейство операторов $\left\{a_{\boldsymbol{k}} \in \mathscr{L}(H), k \in \mathbb{Z}^{d}\right\}$ с нормой $\left\|a_{\boldsymbol{k}}\right\|=t_{\boldsymbol{k}}$, при этом

1) семейство $\left\{S_{\boldsymbol{n}} / \sqrt{|\boldsymbol{n}|}\right\}_{\boldsymbol{n} \in \mathbb{N}^{d}}$ не плотно;

2) существует последовательность $\left\{\boldsymbol{n}_{k}\right\}_{k \in \mathbb{N}} \subset \mathbb{Z}^{d}$ mакая, ито $\boldsymbol{n}_{k} \rightarrow \infty u$ $\mathrm{E}\left\|S_{\boldsymbol{n}_{k}}\right\|^{2} /\left|\boldsymbol{n}_{k}\right| \rightarrow \infty$ npu $k \rightarrow \infty$

3) семейство $\left\{S_{\boldsymbol{n}} / \sqrt{\mathrm{E}\left\|S_{\boldsymbol{n}}\right\|^{2}}\right\}_{\boldsymbol{n} \in \mathbb{N}^{d}}$ не плотно.

ДокАЗАТЕЛьСТво. Для доказательства построим указанные вьше множества случайных величин и операторов. Положим

$$
\begin{gathered}
T_{\boldsymbol{n}}=\sum_{\boldsymbol{k} \in[-\boldsymbol{n}, \boldsymbol{n}]} t_{\boldsymbol{k}}, \quad \boldsymbol{n} \in \mathbb{N}^{d}, \quad z_{k}=\left(1+T_{([\sqrt[2 d]{k} / 3], \ldots,[\sqrt[2 d]{k} / 3])}\right)^{-1}, \quad k \in \mathbb{N}, \\
g_{p}=\sqrt{z_{p}-z_{p+1}}, \quad p \in \mathbb{N} .
\end{gathered}
$$

Пусть $\left\{N_{p, \boldsymbol{k}}\right\}_{p \in \mathbb{N}, \boldsymbol{k} \in \mathbb{Z}^{d}}-$ массив н.о.р. действительных случайных величин, имеющих стандартное нормальное распределение, и $\left\{e_{k}\right\}_{p=1}^{\infty}-$ ортонормированньй базис пространства $H$. Определим действие линейных операторов $\left\{a_{\boldsymbol{k}}\right\}$ на базисных вектоpax:

$$
a_{\boldsymbol{k}}\left(e_{p}\right)=t_{\boldsymbol{k}} e^{-\|\boldsymbol{k}\|^{2} / p} e_{p}=a_{\boldsymbol{k}}(p) e_{p}, \quad k \in \mathbb{Z}^{d}, \quad p \geqslant 1
$$

где $\|\boldsymbol{k}\|=\sqrt{k_{1}^{2}+\cdots+k_{d}^{2}}$. Очевидно, что $\left\|a_{\boldsymbol{k}}\right\|_{\mathscr{L}(H)}=t_{\boldsymbol{k}}$.

Определим теперь случайные величины

$$
\xi_{\boldsymbol{k}}=\sum_{p=1}^{\infty} g_{p} N_{p, \boldsymbol{k}} e_{p}
$$


Легко заметить, что $\mathrm{E} \xi_{\boldsymbol{k}}=0$ и $0<\mathrm{E}\left\|\xi_{\mathbf{0}}\right\|^{2}<\infty$, так как

$$
\mathrm{E}\left\|\xi_{\mathbf{0}}\right\|^{2}=\sum_{p=1}^{\infty} g_{p}^{2} \mathrm{E} N_{p, \mathbf{0}}^{2}=\sum_{p=1}^{\infty} g_{p}^{2}=z_{1}=\frac{1}{1+T_{[\mathbf{1} / 3]}}=\frac{1}{1+T_{\mathbf{0}}}=\frac{1}{1+t_{\mathbf{0}}}
$$

Тогда

$$
\begin{aligned}
S_{\boldsymbol{n}} & =\sum_{\boldsymbol{1} \leqslant \boldsymbol{k} \leqslant \boldsymbol{n}} X_{\boldsymbol{k}}=\sum_{\mathbf{1} \leqslant \boldsymbol{k} \leqslant \boldsymbol{n}} \sum_{\boldsymbol{j} \in \mathbb{Z}^{d}} a_{\boldsymbol{j}}\left(\xi_{\boldsymbol{k}-\boldsymbol{j}}\right) \\
& =\sum_{\boldsymbol{1} \leqslant \boldsymbol{k} \leqslant \boldsymbol{n}} \sum_{\boldsymbol{j} \in \mathbb{Z}^{d}} \sum_{p=1}^{\infty} g_{p} N_{p, \boldsymbol{k}-\boldsymbol{j}} a_{\boldsymbol{j}}(p) e_{p}=\sum_{p=1}^{\infty} S_{\boldsymbol{n}}(p) e_{p},
\end{aligned}
$$

где

$$
S_{\boldsymbol{n}}(p)=g_{p} \sum_{\mathbf{1} \leqslant k \leqslant n} \sum_{\boldsymbol{j} \in \mathbb{Z}^{d}} N_{p, \boldsymbol{k}-\boldsymbol{j}} a_{\boldsymbol{j}}(p) .
$$

Заметим, что при любом фиксированном $p \geqslant 1$ операторы $\left\{a_{\boldsymbol{j}}(p)\right\}_{\boldsymbol{j} \in \mathbb{Z}^{d}}$ удовлетворяют условиям теоремы 2 в одномерном пространстве $H^{(p)}=\left\{\lambda e_{p}\right\}_{\lambda \in \mathbb{R}}$. Действительно,

$$
\sum_{\boldsymbol{j} \in \mathbb{Z}^{d}}\left\|a_{\boldsymbol{j}}(p)\right\|_{\mathscr{L}\left(H^{(p)}\right)}=\sum_{\boldsymbol{j} \in \mathbb{Z}^{d}} t_{\boldsymbol{j}} e^{-\left(j_{1}^{2}+\cdots+j_{d}^{2}\right) / p}<\infty .
$$

Следовательно, по теореме 2

$$
\frac{S_{\boldsymbol{n}}(p)}{\sqrt{|\boldsymbol{n}|}} \stackrel{\mathscr{D}}{\rightarrow} \mathscr{N}\left(0, c_{p}\right), \quad \boldsymbol{n} \rightarrow \infty
$$

где $c_{p}$ - некоторые положительные числа.

Рассмотрим множество

$$
\left\{r_{\boldsymbol{n}}=\frac{\mathrm{E}\left\|S_{\boldsymbol{n}}\right\|^{2}}{|\boldsymbol{n}|}, \boldsymbol{n} \geqslant \mathbf{1}\right\} .
$$

Выберем последовательность $\boldsymbol{n}_{k} \in \mathbb{N}^{d}, \boldsymbol{n}_{k}=(k, \ldots, k), k \in \mathbb{N}$, и докажем, что

$$
r_{\boldsymbol{n}_{k}} \rightarrow \infty \quad \text { при } k \rightarrow \infty \text {. }
$$

В самом деле,

$$
\begin{aligned}
r_{\boldsymbol{n}_{k}} & =\frac{\mathrm{E} \|\left. S_{\boldsymbol{n}_{k}}\right|^{2}}{\left|\boldsymbol{n}_{k}\right|} \geqslant \frac{1}{\left|\boldsymbol{n}_{k}\right|} \sum_{p=\left|\boldsymbol{n}_{k}\right|^{2}}^{\infty} \mathrm{E} S_{\boldsymbol{n}_{k}}^{2}(p) \\
& =\frac{1}{\left|\boldsymbol{n}_{k}\right|} \sum_{p=\left|\boldsymbol{n}_{k}\right|^{2}}^{\infty} g_{p}^{2} \mathrm{E}\left(\sum_{\boldsymbol{j} \in \mathbb{Z}^{d}} \sum_{\mathbf{1} \leqslant l \leqslant \boldsymbol{n}_{k}} a_{\boldsymbol{j}}(p) N_{p, \boldsymbol{l}-\boldsymbol{j}}\right)^{2} \\
& =\frac{1}{\left|\boldsymbol{n}_{k}\right|} \sum_{p=\left|\boldsymbol{n}_{k}\right|^{2}}^{\infty} g_{p}^{2} \mathrm{E}\left(\sum_{\boldsymbol{j} \in \mathbb{Z}^{d}} \sum_{\mathbf{1} \leqslant l \leqslant \boldsymbol{n}_{k}} a_{\boldsymbol{l}+\boldsymbol{j}}(p) N_{p,-\boldsymbol{j}}\right)^{2} \\
& =\frac{1}{\left|\boldsymbol{n}_{k}\right|} \sum_{p=\left|\boldsymbol{n}_{k}\right|^{2}}^{\infty} g_{p}^{2} \sum_{\boldsymbol{j} \in \mathbb{Z}^{d}}\left(\sum_{\mathbf{1}+\boldsymbol{j} \leqslant l \leqslant \boldsymbol{n}_{k}+\boldsymbol{j}} t_{\boldsymbol{l}} e^{-\|\boldsymbol{l}\|^{2} / p}\right)^{2} .
\end{aligned}
$$


Куб $\Delta_{j}=\left\{\boldsymbol{l} \in \mathbb{Z}^{d}: \mathbf{1}+\boldsymbol{j} \leqslant \boldsymbol{l} \leqslant \boldsymbol{n}_{k}+\boldsymbol{j}\right\}$ включает в себя меньший куб $\Delta=\left\{\boldsymbol{m} \in \mathbb{Z}^{d}\right.$ : $-\left[\boldsymbol{n}_{k} / 3\right] \leqslant \boldsymbol{m} \leqslant\left[\boldsymbol{n}_{k} / 3\right\}$, если вьполняются неравенства

$$
\mathbf{1}+\boldsymbol{j} \leqslant-\left[\frac{\boldsymbol{n}_{k}}{3}\right], \quad \boldsymbol{n}_{k}+\boldsymbol{j} \geqslant\left[\frac{\boldsymbol{n}_{k}}{3}\right] .
$$

В координатной записи это означает, что

$$
\left[\frac{k}{3}\right]-k \leqslant j_{i} \leqslant-\left[\frac{k}{3}\right]-1, \quad i=1, \ldots, d .
$$

Таким образом, вектор $\boldsymbol{j}$ может принимать по каждой координате ровно

$$
\left(\left(-\left[\frac{k}{3}\right]-1\right)-\left(\left[\frac{k}{3}\right]-k\right)+1\right)=k-2\left[\frac{k}{3}\right]
$$

значений. Так как $k-2[k / 3] \geqslant k / 3$, то всего различных $j$, удовлетворяюших системе (19), будет не менее $(k / 3)^{d}=\left|\boldsymbol{n}_{k}\right| / 3^{d}$. Учитьвая это, продолжим неравенство (18):

$$
\begin{aligned}
& r_{\boldsymbol{n}_{k}} \geqslant \frac{1}{\left|\boldsymbol{n}_{k}\right|} \sum_{p=\left|\boldsymbol{n}_{k}\right|^{2}}^{\infty} g_{p}^{2} \cdot\left|\frac{\boldsymbol{n}_{k}}{3}\right| \cdot\left(\sum_{-\left[\boldsymbol{n}_{k} / 3\right] \leqslant \boldsymbol{l} \leqslant\left[\boldsymbol{n}_{k} / 3\right]} t_{\boldsymbol{l}} e^{-\|\boldsymbol{l}\|^{2} / p}\right)^{2} \\
& \geqslant \frac{\left|\boldsymbol{n}_{k} / 3\right|}{\left|\boldsymbol{n}_{k}\right|} \cdot\left\{e^{-\left|\left[\boldsymbol{n}_{k} / 3\right]\right|^{2} /\left|\boldsymbol{n}_{k}\right|^{2}}\right\}^{2} \cdot z_{\left|\boldsymbol{n}_{k}\right|^{2}} \cdot T_{\left[\boldsymbol{n}_{k} / 3\right]}^{2} \\
& \geqslant \frac{1}{3^{d}} e^{-2 / 9^{d}} \cdot \frac{T_{([k / 3], \ldots,[k / 3])}^{2}}{1+T_{\left(\left[\sqrt[2 d]{\left|\boldsymbol{n}_{k}\right|^{2}} / 3\right], \ldots,\left[\sqrt[2 d]{\left|\boldsymbol{n}_{k}\right|^{2}} / 3\right]\right)}} \\
& =\frac{1}{3^{d}} e^{-2 / 9^{d}} \cdot \frac{T_{([k / 3], \ldots,[k / 3])}^{2}}{1+T_{([k / 3], \ldots,[k / 3])}} \rightarrow \infty, \quad k \rightarrow \infty \text {. }
\end{aligned}
$$

Таким образом, доказали (17) и утверждение 2) теоремы 3.

Очевидно, что

$$
\frac{S_{\boldsymbol{n}_{k}}}{\sqrt{\mathrm{E}\left\|S_{\boldsymbol{n}_{k}}\right\|^{2}}}=\frac{S_{\boldsymbol{n}_{k}}}{\sqrt{\left|\boldsymbol{n}_{k}\right|}} \cdot \sqrt{\frac{\left|\boldsymbol{n}_{k}\right|}{\mathrm{E} \| S_{\boldsymbol{n}_{k} \|^{2}}}} .
$$

Обозначим символом $\theta$ случайную величину $\theta: \Omega \rightarrow H$, принимающую тождественно нулевое значение. Напомним, что конечномерными распределениями случайного элемента $\zeta: \Omega \rightarrow H$ называется семейство распределений проекций $\zeta$ на конечномерные подпространства $H^{k} \subset H$. Тогда из (14)-(17) и (20) следует, что верно утверждение

A: все конечномерные распределения последовательности $\left\{S_{\boldsymbol{n}_{k}} / \sqrt{\mathrm{E}\left\|S_{\boldsymbol{n}_{k}}\right\|^{2}}\right\}_{k \in \mathbb{N}}$ сходятся к конечномерным распределениям величины $\theta$.

Предположим, что выполнено также предположение

В: последовательность $\left\{S_{\boldsymbol{n}_{k}} / \sqrt{\mathrm{E}\left\|S_{\boldsymbol{n}_{k}}\right\|^{2}}\right\}_{k \in \mathbb{N}}$ плотна. 
Тогда в силу А и В она сходится по распределению к нулю как элементу гильбертова пространства $H$ (см. [5, гл. 2.1]).

Рассмотрим последовательность $Y_{k}=\left\|S_{\boldsymbol{n}_{k}}\right\|^{2} / \mathrm{E}\left\|S_{\boldsymbol{n}_{k}}\right\|^{2}$ и докажем, что она равномерно интегрируема. Действительно,

$$
\begin{aligned}
\lim _{c \rightarrow \infty} \sup _{k} \mathrm{E} Y_{k} I\left\{Y_{k}>c\right\} & =\lim _{c \rightarrow \infty} \sup _{k} \frac{1}{\mathrm{E}\left\|S_{\boldsymbol{n}_{k}}\right\|^{2}} \mathrm{E}\left\|S_{\boldsymbol{n}_{k}}\right\|^{2} I\left\{\left\|S_{\boldsymbol{n}_{k}}\right\|^{2}>c \mathrm{E}\left\|S_{\boldsymbol{n}_{k}}\right\|^{2}\right\} \\
& \leqslant \lim _{c \rightarrow \infty} \sup _{k} \frac{1}{\left(\mathrm{E}\left\|S_{\boldsymbol{n}_{k}}\right\|^{2}\right)^{2}} \cdot \frac{1}{c} \mathrm{E}\left\|S_{\boldsymbol{n}_{k}}\right\|^{4} .
\end{aligned}
$$

Так как

$$
\left\|S_{\boldsymbol{n}_{k}}\right\|^{2}=\sum_{\boldsymbol{j} \in \mathbb{Z}^{d} \times \mathbb{N}} v_{j}^{2} \eta_{\boldsymbol{j}}^{2},
$$

где $\eta_{\boldsymbol{j}} \sim \mathscr{N}(0,1)$, независимы, а $v_{\boldsymbol{j}}^{2} \geqslant 0$, то

$$
\begin{aligned}
\mathrm{E}\left\|S_{\boldsymbol{n}_{k}}\right\|^{4} & =\mathrm{E}\left(\sum_{\boldsymbol{j} \in \mathbb{Z}^{d} \times \mathbb{N}} v_{\boldsymbol{j}}^{2} \eta_{\boldsymbol{j}}^{2}\right)^{2}=\sum_{\boldsymbol{j} \in \mathbb{Z}^{d} \times \mathbb{N}} v_{\boldsymbol{j}}^{4} \mathrm{E} \eta_{\boldsymbol{j}}^{4}+2 \sum_{\boldsymbol{i} \neq \boldsymbol{j}} v_{\boldsymbol{i}}^{2} v_{\boldsymbol{j}}^{2} \mathrm{E} \eta_{\boldsymbol{i}}^{2} \mathrm{E} \eta_{\boldsymbol{j}}^{2} \\
& \leqslant 3\left(\sum_{\boldsymbol{j} \in \mathbb{Z}^{d} \times \mathbb{N}} v_{\boldsymbol{j}}^{2}\right)^{2}=3\left(\mathrm{E}\left\|S_{\boldsymbol{n}_{k}}\right\|^{2}\right)^{2}
\end{aligned}
$$

и можно продолжить неравенство (21). Получим

$$
\lim _{c \rightarrow \infty} \sup _{k} \mathrm{E} Y_{k} I\left\{Y_{k}>c\right\} \leqslant \lim _{c \rightarrow \infty} \sup _{k} \frac{3}{c}=0 .
$$

Так как $Y_{k} \stackrel{\mathscr{D}}{\rightarrow} 0, k \rightarrow \infty$, и $\left\{Y_{k}\right\}-$ равномерно интегрируемы, то по теореме 5.4 из [6] получаем $\mathrm{E} Y_{k} \rightarrow \mathrm{E} 0=0$. Но $\mathrm{E} Y_{k}=1 \nrightarrow 0$. Получили противоречие. Значит, предположение В неверно и последовательность $\left\{S_{\boldsymbol{n}_{k}} / \sqrt{\mathrm{E}\left\|S_{\boldsymbol{n}_{k}}\right\|^{2}}\right\}_{k \in \mathbb{N}}$ не плотна, тогда и все семейство $\left\{S_{\boldsymbol{n}} / \sqrt{\mathrm{E}\left\|S_{\boldsymbol{n}}\right\|^{2}}\right\}_{\boldsymbol{n} \in \mathbb{Z}^{d}}$ не плотно. Этим доказано утверждение 3$)$ теоремы 3.

Далее, из (20) легко видеть, что последовательность $\left\{S_{\boldsymbol{n}_{k}} / \sqrt{\left|\boldsymbol{n}_{k}\right|}\right\}_{k \in \mathbb{N}}$ такженеплотна, что влечет утверждение 1). Теорема 3 доказана.

Автор выражает благодарность проф. А. В. Булинскому за постановку задачи и внимание к работе.

\section{СПИСОК ЦИТИРОВАННОЙ ЛИТЕРАТУРЫ}

[1] Merlevède F. Sur l'inversibilité des processus linéaires à valeurs dans un espace de Hilbert // Compt. Rend. Acad. Sci. Paris. Sér. 1. 1995. V. 321. P. 477-480.

[2] Merlevède F., Peligrad M., Utev S. Sharp conditions for the CLT of linear processes in a Hilbert space // J. Theor. Probab. 1997. V. 10. № 3. P. 681-693.

[3] Araujo A., Giné E. The Central Limit Theorem for Real and Banach Valued Random Variables. New York: John Wiley and Sons, 1980.

[4] Ибрагимов И.А., Линник Ю. В. Независимые и стационарно связанные случайные величины. М.: Наука, 1965.

[5] Ledoux M., Talagrand M. Probability in Banach Space. Berlin: Springer, 1991.

[6] Биллингсли П. Сходимость вероятностных мер. М.: Наука, 1977. 